\title{
Identifying stably expressed genes from multiple RNA-Seq data sets
}

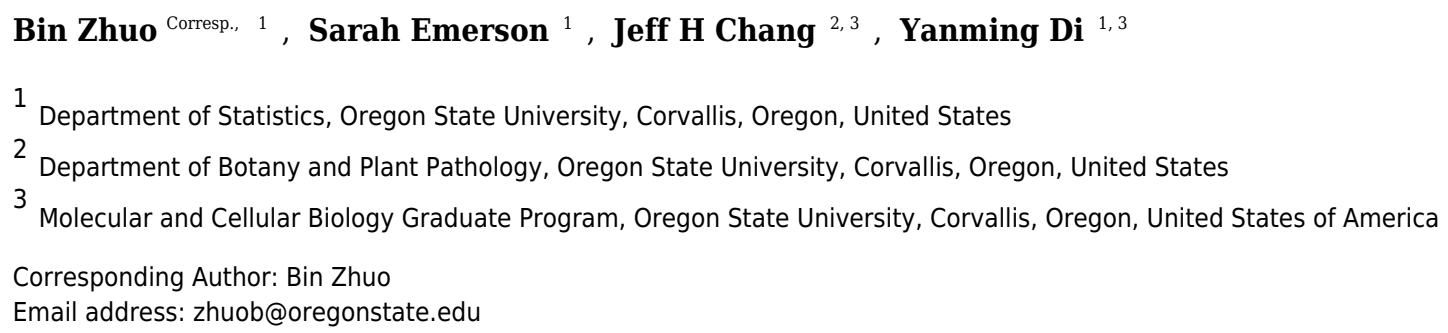

We examined RNA-Seq data on 211 biological samples from 24 different Arabidopsis experiments carried out by different labs. We grouped the samples according to tissue types, and in each of the groups, we identified genes that are stably expressed across biological samples, treatment conditions, and experiments. We fit a Poisson log-linear mixed-effect model to the read counts for each gene and decomposed the total variance into between-sample, between-treatment and between-experiment variance components. Identifying stably expressed genes is useful for count normalization and differential expression analysis. The variance component analysis that we explore here is a first step towards understanding the sources and nature of the RNA-Seq count variation. When using a numerical measure to identify stably expressed genes, the outcome depends on multiple factors: the background sample set and the reference gene set used for count normalization, the technology used for measuring gene expression, and the specific numerical stability measure used. Since differential expression (DE) is measured by relative frequencies, we argue that $\mathrm{DE}$ is a relative concept. We advocate using an explicit reference gene set for count normalization to improve interpretability of DE results, and recommend using a common reference gene set when analyzing multiple RNA-Seq experiments to avoid potential inconsistent conclusions. 


\title{
Identifying stably expressed genes from multiple RNA-Seq data sets
}

\author{
Bin Zhuo ${ }^{1}$, Sarah C. Emerson ${ }^{1}$, Jeff H. Chang ${ }^{2,3}$, and Yanming $\mathrm{Di}^{1,2, *}$ \\ ${ }^{1}$ Department of Statistics, Oregon State University, Corvallis, Oregon, United States of \\ America \\ ${ }^{2}$ Molecular and Cellular Biology Program, Oregon State University, Corvallis, Oregon, \\ United States of America, \\ ${ }^{3}$ Department of Botany and Plant Pathology, Oregon State University, Corvallis, Oregon, \\ United States of America, \\ *diy@oregonstate.edu
}

\begin{abstract}
We examined RNA-Seq data on 211 biological samples from 24 different Arabidopsis experiments carried out by different labs. We grouped the samples according to tissue types, and in each of the groups, we identified genes that are stably expressed across biological samples, treatment conditions, and experiments. We fit a Poisson log-linear mixed-effect model to the read counts for each gene and decomposed the total variance into between-sample, between-treatment and between-experiment variance components. Identifying stably expressed genes is useful for count normalization and differential expression analysis. The variance component analysis that we explore here is a first step towards understanding the sources and nature of the RNA-Seq count variation. When using a numerical measure to identify stably expressed genes, the outcome depends on multiple factors: the background sample set and the reference gene set used for count normalization, the technology used for measuring gene expression, and the specific numerical stability measure used. Since differential expression (DE) is measured by relative frequencies, we argue that $D E$ is a relative concept. We advocate using an explicit reference gene set for count normalization to improve interpretability of DE results, and recommend using a common reference gene set when analyzing multiple RNA-Seq experiments to avoid potential inconsistent conclusions.
\end{abstract}

\section{INTRODUCTION}

RNA sequencing (RNA-Seq) has become the technology of choice for transcriptome profiling over the last few years. The exponential growth in RNA-Seq studies have produced a large amount of Arabidopsis thaliana (Arabidopsis) data under a variety of experimental/environmental conditions. It is only natural to begin exploring how the large amount of existing data sets can help the analysis of future data. In this paper, we discuss identifying stably expressed genes from multiple existing RNA-Seq data sets based on a numerical measure of stability. We envision that such identified stably expressed genes could be used as a reference set or prior information for count normalization and differential expression (DE) analysis of future RNA-Seq data sets obtained from similar or comparable experiments. We also fit a random-effect model to the read counts for each gene and decompose the total variance into between-sample, betweentreatment and between-experiment variance components. The variance component analysis is a first step towards understanding the sources and nature of the RNA-Seq count variation. To illustrate our methods, we examined RNA-Seq data on 211 Arabidopsis samples from 24 different experiments carried out by different labs and identified genes that were stably expressed across biological samples, experimental or environmental conditions, and experiments (labs).

A reference set of stably-expressed genes will be useful for count normalization. A key task of RNA-Seq analysis is to detect DE genes under various experimental or environmental conditions. Count normalization is needed to adjust for differences in sequencing depths or library sizes (total numbers of mapped reads for each biological sample) due to chance variation in sample preparation. In DE analysis, 
gene expression levels are often estimated from relative read frequencies. For this reason, normalization is also needed to account for the fact that non-differentially expressing genes may exhibit an apparent reduction or increase in relative read frequencies due to the respective increased or decreased relative read frequencies of truly differentially expressing genes. Many existing normalization methods, such as the trimmed mean of M-values normalization method (TMM) (Robinson and Oshlack, 2010) and Anders and Huber's normalization (Anders and Huber, 2010), assume that the majority of the genes within an experiment are not $\mathrm{DE}$, and examine the sample distribution of the fold changes between samples. If the experiment condition can affect expression levels of more than half of the genes, many of the existing normalization methods may be unreliable (Lovén et al., 2012; Wu et al., 2013). This difficulty could be alleviated if one could identify a set of stably expressed genes whose expression levels are known or expected to not vary much under different experimental conditions. Our idea is to identify such a reference set based on a large number of existing data sets.

Our basic intuition is that a numerical quantification of expression stability-which typically measures certain aspects of RNA-Seq count variation — can be more reliably estimated by using more data sets. There is, however, a caveat to this idea: as pointed out by Fernandes et al. (2008) and Hruz et al. (2011), universally stably expressed genes may not exist. Hruz et al. showed that a subset of stably expressed genes from a specific biological context may have more variability than other genes if examined across a broader range of samples and conditions. Many studies have shown that stably expressed genes are subject to change from one experiment to another due to different experimental protocols, different tissue types, or other varying conditions (Hong et al., 2010; Reid et al., 2006). The top 100 stably expressed genes in the Arabidopsis developmental series of Czechowski et al. (2005) shared only 3 genes with the top 50 stably expressed genes identified from Arabidopsis seed samples by Dekkers et al. (2012). In this study, we try to balance generality and specificity by identifying different reference gene sets for different tissue types of Arabidopsis.

We can also consider that when a normalization method is applied to a single data set, it effectively specifies an implicit reference set of stably expressed genes (those genes that have the least variation after normalization). From this perspective, we can view commonly used normalization techniques as using an internally identified reference set of genes. In contrast, what we are proposing is that one could alternatively identify a reference set externally by looking at past data sets. The internally and externally identified reference gene sets will provide different contexts for the DE analysis: in other words, one can choose to answer different scientific questions by using different reference sets. In any case, we advocate making the reference set explicit during a DE analysis and using a common reference set when analyzing multiple datasets.

We want to clarify that having stable gene expression is not equivalent to maintaining a stable biological function. Often times, we may not understand the biological functions of genes with numerically stable expression measures. From an operational point of view, however, numerical stability is more tractable. In the pre-genomic era, the so-called "house-keeping genes" were often considered to be candidate reference genes for normalization (Andersen et al., 2004; Bustin, 2002). House-keeping genes are typically constitutive genes that maintain basic cellular function, and therefore are expected to express at relatively constant levels in non-pathological situations. However, many studies have shown that house-keeping genes are not necessarily stably expressed according to numerical measures (a review can be found in Huggett et al. (2005) and reference therein). For example, in the microarray analysis of Arabidopsis, Czechowski et al. showed that traditional house-keeping genes such as ACT2, TUB6, EF-1 $\alpha$ are not stably expressed, and thus not good reference genes for normalization. Spike-in genes have also been considered as reference genes for normalization, but Risso et al. (2014) showed that spike-in genes are not necessarily stably expressed according numerical measures either.

In this paper, we identify stably expressed genes from RNA-Seq data sets based on a numerical measure - the sum of three variance components estimated from a mixed-effect model. For microarray data, there have been many efforts to numerically find stably expressed genes by quantifying the variation of measured expression levels across a large number of microarray data sets. For example, Andersen et al. (2004) used a linear mixed model to estimate the between-group and within-group variances from expression profiles of microarray experiments, and then quantified expression stability by combining the two variance components using a Bayesian formulation. Czechowski et al. measured the expression stability of each gene using the coefficient of variation $(\mathrm{CV})$. Genes with lower $\mathrm{CV}$ s are considered more stably expressed. By investigating 721 arrays under 323 conditions throughout development, Czechowski 


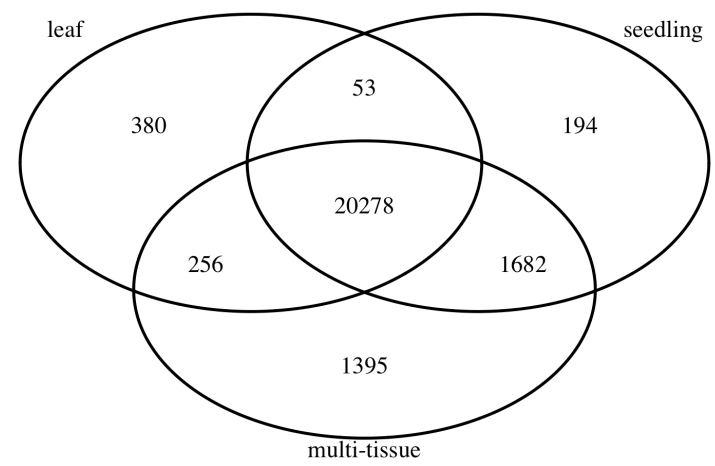

Figure 1. The numbers and overlap of the genes in the three groups of Abrabidopsis samples after removing genes with low mean counts.

et al. suggested stably expressed (reference) genes under different experimental conditions for Arabidopsis. Stamova et al. (2009), Dekkers et al., Gur-Dedeoglu et al. (2009), and Frericks and Esser (2008) screened a large number of microarray data sets to identify stably expressed genes in human blood, Arabidopsis seed, breast tumor tissues, and mice respectively. Validation experiments (Czechowski et al., 2005; Dekkers et al., 2012; Huggett et al., 2005; Stamova et al., 2009) showed that these genes are more stably expressed than traditional house-keeping genes.

Our vision is that identifying stably expressed genes is the first step towards integrative analysis of multiple RNA-Seq experiments. It will help to answer fundamental questions related to comparability, reproducibility and replicability of RNA-Seq experiments.

\section{MATERIALS \& METHODS}

In Section 2.1, we describe the steps for collecting and processing RNA-Seq data sets from Arabidopsis experiments. In Section 2.2, we discuss count normalization methods and how to apply them to a subset of stably expressed genes. In Section 2.3, we introduce the generalized linear mixed model (GLMM, McCulloch and Neuhaus 2001) for estimating three variance components from RNA-Seq data: the between-sample, between-treatment and between-experiment variances. We define the total variance measure for expression stability as the sum of estimated variance components. In Section 2.4, we review the $\mathrm{CV}$ and $\mathrm{M}$-value measures for gene expression stability.

\subsection{RNA-Seq data collection and processing} 2.1.1 Overview of the RNA-Seq data sets

We examined RNA-Seq data from 49 Arabidopsis experiments stored on the NCBI GEO repository (see more details below). After screening, we retained data from 211 biological samples in 24 experiments. To illustrate our methods for finding stably expressed genes, we divided the experiments into three groups: the seedling group contains 60 Arabidopsis seedling samples from 9 experiments; the leaf group contains 60 Arabidopsis leaf samples from 5 experiments; the multi-tissue group contains 91 samples from 10 experiments on multiple tissue types (shoot apical, root tip, primary root, inflorescences and siliques, hypocotyl, flower, carpels, aerial tissue, epidermis, seed). Table 1 summarizes the basic information about the three groups (see Supplemental Table S1 for more details).

Table 1. Summary statistics for the three groups of Arabidopsis samples.

\begin{tabular}{lllll}
\hline Group & \# experiments & \# treatments & \# samples & \# genes \\
\hline seedling & 9 & 27 & 60 & 22207 \\
leaf & 5 & 28 & 60 & 20967 \\
multi-tissue & 10 & 39 & 91 & 23611 \\
\hline
\end{tabular}

To find stably expressed genes in each group, we processed the raw sequencing data and summarized the results as count matrices of mapped RNA-Seq short reads (see details below). We removed genes with 
low mean numbers (less than 3) of mapped read counts for all experiments. Such genes tend to be more prone to sequencing noise, less interesting to biologists, and also cause convergence issues when fitting statistical models. Many other researchers (such as Anders et al. 2013) recommend removing such genes before analyzing RNA-Seq data. The number of remaining genes in each group is also summarized in Table 1. Figure 1 shows the numbers and overlap of the genes after this step.

\subsubsection{Details of the data processing steps}

The Gene Expression Omnibus (GEO) repository at National Center for Biotechnology Information (NCBI, http://www.ncbi.nlm.nih.gov/) stores raw sequencing data from a large number of RNA-Seq experiments. For this study, we restrict our attention to Arabidopsis experiments satisfying the following conditions: 1 . Ecotype $=$ "Columbia" (we kept only the Columbia samples from experiments that compare Columbia samples to other ecotypes); 2 . There are at least two treatments and 2 biological replicates for each treatment; 3 . Library strategy $=$ "RNA-Seq"; 4. Library source = "transcriptomic"; 5 . Library selection= "cDNA"; 6. Library layout = "Single end"; 7. If there are repeated measurements over time, we choose samples from one time point. We screened all the Arabidopsis experiments available from the NCBI GEO repository up to May 31, 2015 and downloaded raw RNA-Seq data (Sequence Read Archive files) from 49 experiments.

We assembled our own in-house pipeline to process all the raw RNA-Seq data: align the raw RNA-Seq reads to the reference genome and summarize the read counts at the gene level. In the GEO repository, the mapped read counts are unavailable for some experiments and the available ones are from different processing pipelines. Our pipeline, implemented using the software R (R Core Team, 2015), is summarized as follows:

1. Convert the Sequence Read Archive (SRA) files to FASTQ files using the NCBI SRA Toolkit (Leinonen et al. (2010), version 2.3.5-2).

2. Download the reference genome

Arabidopsis_thaliana.TAIR10.22.dna.toplevel.fa

from the Ensembl plants FTP server (http://plants.ensembl.org/info/data/ftp/ index.html) and build index using build ( ) function from Subread aligner (RSubread, version 1.16.2, Liao et al. 2013) in the software R (R Core Team, 2015). The index allows fast retrieval of the sets of positions in the reference genome where the short reads are more likely to align.

3. Align short reads in FASTQ files to the Arabidopsis reference genome using the align () function from Rsubread.

4. Summarize the read counts at the gene level using the featureCounts () function from the Subread aligner and store the read counts as data matrix. The annotation file

Arabidopsis_thaliana.TAIR10.22.gtf

is downloaded from Ensembl plants FTP server. To keep the pipeline simple, we did not count multi-mapping or multi-overlapping reads. One potential challenge when dealing with multimapping reads is that existing methods will assign reads to different gene features proportionally and probabilistically, it is unclear to us how to handle the additional uncertainly associated with such a process (see, e.g., Anders et al. (2014)). DE analysis of multiple mapped reads often requires special method.

Subread aligner is a recently developed sequence mapping tool that adopts a seed-and-vote paradigm to map the RNA-Seq short reads to the genome. It breaks each short read into a series of overlapping segments called subreads and uses the subreads to vote on the optimal genome location of the original read. The subreads are shorter and can be mapped to the genome much faster. Compared to other aligners such as Bowite 2 (Langmead and Salzberg, 2012) or BWA (Li and Durbin, 2009), Subread aligner is both faster and more accurate (Liao et al., 2013). We compared results from the above pipeline to results from a pipeline described in Anders et al. (2013) over several RNA-Seq experiment data, and Rsubread was 
more than three times faster and successfully mapped more reads to the reference genome. For researchers familiar with R, it also has the advantage that it is completely implemented in R.

We divided the experiments into three groups as summarized in Table 1. As an additional data quality control measure, we keep an experiment only when it has mapping quality (number of successfully mapped reads divided by total number of reads) $\geq 50 \%$ for all samples. Then within each group, we computed an initial set of normalization factors from all samples combined using the method described in Section 2.2. An experiment is retained only when the normalization factors of all samples in the experiment are between 0.50 and 1.50. If the initial estimated normalization factor is too different from 1 for a sample, it often indicates that the read counts distribution in the corresponding sample is markedly different from the distributions of the rest of the samples. Such samples demand additional attention before being incorporated in the studies that we intend to do.

\subsection{Count normalization}

As explained in the introduction, count normalization is needed when analyzing RNA-Seq data to 1) adjust for differences in sequencing depths or library sizes; 2) to adjust for the apparent changes in relative read frequencies of non-DE genes that occur as a consequence of changes in relative read frequencies of truly DE genes.

For the second type of adjustment, we follow Anders and Huber's method (Anders and Huber, 2010) for estimating normalization factors. Let $y_{i j}$ denote the read count for $i$ th gene of the $j$ th sample ( $m$ genes and $n$ samples in total). We first create a pseudo-reference sample where each gene's expression value is the geometric mean expression over all real samples for that gene,

$$
y_{i, 0}=\left(\prod_{j=1}^{n} y_{i, j}\right)^{1 / n}, i=1, \ldots, m .
$$

Next we calculate the median fold-change in relative frequency between each sample $j$ and the pseudoreference sample,

$$
R_{j}^{\prime}=\operatorname{median}\left(\frac{y_{1, j} / N_{j}}{y_{1,0} / N_{0}}, \ldots, \frac{y_{m, j} / N_{j}}{y_{m, 0} / N_{0}}\right),
$$

where $N_{j}$ is the library size for sample $j$ (the sum of RNA-Seq counts mapped to all genes retained in each sample). Finally, the normalization factor $R_{j}$ for sample $j$ is calculated as

$$
R_{j}=\frac{R_{j}^{\prime}}{\left(\prod_{j=1}^{n} R_{j}^{\prime}\right)^{1 / n}} .
$$

Using the estimated normalization factors, the relative frequencies will be computed as $y_{i j} / N_{j} R_{j}$, which we will call the normalized relative frequency for gene $i$ in sample $j$. The assumption made here is that the median fold change between normalized relative frequencies in two samples should be 1 . In other words, this normalization method assumes that the majority of genes are not DE. The NBPSeq package (Di et al., 2014) has an inbuilt function for this procedure and it will be used for count normalization in this paper. With the estimates from equation (3), we see that the median fold change in normalized relative frequencies between each sample and the pseudo-reference sample will be set to 1 :

$$
\operatorname{median}\left(\frac{y_{1, j} / N_{j} R_{j}}{y_{1,0} / N_{0} R_{0}}, \ldots, \frac{y_{m, j} / N_{j} R_{j}}{y_{m, 0} / N_{0} R_{0}}\right)=1,
$$

where $R_{0}=\left(\prod_{j=1}^{n} R_{j}^{\prime}\right)^{-1 / n}$.

We can apply equation (2) to a subset of reference genes to estimate normalization factors. In doing so, effectively, the median fold change in equation (4) among the reference genes will be set to 1 in each sample $j$. Other normalization methods may make different assumptions than Anders and Huber's, but some assumptions of a similar nature seem unavoidable. For example, the TMM method of Robinson and Oshlack (2010) is based on a similar principle: assuming the majority of the genes are not DE. The TMM method can be applied to a subset of genes selected based on an initial screening of mean expression level and fold changes. In TMM method, one can also specify certain quantile (instead of the median) of the fold changes to be 1 . 
In this paper, we will identify stably expressed genes from multiple data sets based on numerical measure and use them as reference for estimating normalization factors (from equations (2) and (3)). However, to identify the stably expressed genes, we first need a set of initially estimated normalization factors. To tackle this circular dependence, we use a one-step iteration method to estimate the normalization factors:

1. First, we use all the genes to calculate the initial normalization factors;

2. Then, we fit a GLMM to each gene and estimate the total variance measure, incorporating the initial normalization factors as an offset term (see Section 2.3);

3. Next, we select the top 1000 stably expressed genes based on the total variance measure estimated from step 2 above, and use them as reference genes to recalculate the normalization factors.

In practice, this one-step method seems to be adequate and further iterations will only slightly change the set of 1000 stably expressed genes. For example, for the multi-tissue group of experiments, if we were to run one more iteration of steps 2 and 3, there would be 946 overlapping genes between the top 1000 genes from the first iteration and those from the second iteration.

\subsection{Poisson log-linear mixed-effects regression model and the total variance measure of expression stability}

We fit a Poisson log-linear mixed-effects regression model to the RNA-Seq counts mapped to each gene and measure gene expression stability using a total variance measure. Let $Y_{i j k l}$ be the number of RNA-Seq reads mapped to gene $i$ in sample $j$ from treatment group $k$ in experiment $l$. We will fit regression models to each gene separately and suppress subscript $i$ from the model equations. For each gene, we fit a Poisson log-linear mixed-effects regression model

$$
\begin{aligned}
& Y_{j k l} \sim \operatorname{Poisson}\left(\mu_{j k l}\right), \\
& \log \left(\mu_{j k l}\right)=\log \left(R_{j k l} N_{j k l}\right)+\xi+\alpha_{l}+\beta_{k(l)}+\varepsilon_{j k l},
\end{aligned}
$$

which is a specific type of generalized linear mixed model (GLMM, McCulloch and Neuhaus (2001)). In equation (6), $N_{j k l}$ and $R_{j k l}$ are the library size and normalization factor discussed in Section 2.2. We will call $R_{j k l} N_{j k l}$ the normalized library size. The parameter $\xi$ is a fixed-effect term for the baseline log mean of the relative counts (counts divided by the normalized library sizes). The values $\alpha, \beta$, and $\varepsilon$ represent the experiment effect, the treatment effect (nested within each experiment), and the sample effect respectively. We view $\alpha, \beta$ and $\varepsilon$ as random effects and assume that they are independent and follow normal distributions:

$$
\alpha_{l} \sim N\left(0, \sigma_{\text {experiment }}^{2}\right), \beta_{k(l)} \sim N\left(0, \sigma_{\text {treatment }}^{2}\right), \quad \varepsilon_{j k l} \sim N\left(0, \sigma_{\text {sample }}^{2}\right),
$$

where $\sigma_{\text {experiment }}^{2}, \sigma_{\text {treatment }}^{2}$ and $\sigma_{\text {sample }}^{2}$ are called variance-components - they quantify the overall variances of the corresponding random effect terms.

The sample effect $\varepsilon$ represents the extra-Poisson variation in read counts among samples in the same treatment group and $\sigma_{\text {sample }}^{2}$ plays a similar role as the over-dispersion parameter in a negative binomial model (Anders and Huber 2010; Di et al. 2011). The experiment effect, $\alpha$, accounts for all sources of variation at the experiment level, including differences in lab personnel and conditions, day light hours, age of the plants, temperature, sequencing platform, and other unidentified sources. The contributions from these different experiment-level sources are often difficult to separate statistically. We treat the experiment effect $\alpha$ as a random effect because we view the collected experiments as a random sample from the pool of all Arabidopsis RNA-Seq experiments. We also treat the treatment effect $\beta$ as a random effect. In a DE test, $\beta$ is usually considered as a fixed-effect term. Here for evaluation of expression stability, we are not interested in the specific levels of the individual $\beta$ 's and focus more on the overall variation of $\beta$ under a range of treatment types.

We define the stability measure as the estimated total variance,

$$
\hat{\sigma}^{2}=\hat{\sigma}_{\text {sample }}^{2}+\hat{\sigma}_{\text {treatment }}^{2}+\hat{\sigma}_{\text {experiment }}^{2}
$$

The parameters $\left(\xi, \sigma_{\text {experiment }}^{2}, \sigma_{\text {treatment }}^{2}, \sigma_{\text {sample }}^{2}\right)$ are estimated using the $g$ lmer ( ) function of the R package lme 4 (Bates et al. (2012), version 1.1.7), which uses a Gaussian-Hermite quadrature to approximate 
the likelihood function. We rank all the genes according to their values of $\hat{\sigma}^{2}$ in increasing order (smallest first), and consider highly ranked (e.g., top 1000) genes to be stably expressed.

Normal models (equation (7)) are commonly assumed for the random effects in the GLMM settings. The normality assumption is likely a simplification of reality, yet it is a good starting point and should be adequate for finding genes with low total variation-the stably expressed ones.

\subsection{Other stability measures}

The assessment of gene expression stability depends on the specific stability measure used. Czechowski et al. and Dekkers et al. used the coefficient of variation (CV) measure, computed as standard deviation devided by mean, to find stably expressed genes from microarray data.

The $M$-value in geNorm (Vandesompele et al., 2002) is a well-cited measure. For a set of $m_{0}$ genes, the $M$-value measure works as follows: First, the pairwise variation between gene $i_{1}$ and gene $i_{2}$ is calculated as the standard deviation of the log fold changes between their expression levels across all the $n$ samples:

$$
V_{i_{1}, i_{2}}=\text { st.dev }\left\{\log \left(\frac{y_{1, i_{1}}}{y_{1, i_{2}}}\right), \ldots, \log \left(\frac{y_{n, i_{1}}}{y_{n, i_{2}}}\right)\right\} .
$$

Next, the $M$-value for gene $i$ is defined as the average pairwise variation between gene $i$ and all other genes

$$
M_{i}=\frac{\sum_{k \neq i} V_{i, k}}{m_{0}-1} .
$$

In the Results section, we compare the $M$-value to the total variance measure on RNA-Seq data from the multi-tissue group experiments, and compare the stably expressed genes identified from these two measures to those identified from microarray data using the $\mathrm{CV}$ measure.

\section{RESULTS}

In Section 3.1, we summarize the stably expressed genes identified from three different experiment groups and emphasize that stability is context-dependent. In Section 3.2, we show that traditional house-keeping genes are not necessarily stably expressed according to our numerical measure, and that microarray data and RNA-Seq data may often give different sets of stably expressed genes. In Section 3.3, we further demonstrate that when using a numerical measure to quantify gene expression stability, the outcome will depend on the specific numeric measure used. These points should be intuitive, but they are not often emphasized in practice. In Section 3.4, we discuss results from our variance component analysis. In Section 3.5, we discuss how to use the identified stably expressed genes for count normalization.

\subsection{Stably Expressed Genes}

Using the total variance, $\hat{\sigma}^{2}$, from the GLMM (see equation (6) in Section 2.3) as a stability measure, we identified stably expressed genes from the three groups of experiments described in Section 2.1: the group of seedling experiments, the group of leaf experiments, and the group of experiments on different tissue types (see Table 1 for a summary). As we mentioned in the Introduction, absolutely stably expressed genes may not exist. Choosing different sample sets as reference allows us to identify stably expressed genes for different biological contexts.

In Supplemental Tables S2-S4, we summarize the top 1000 most stably expressed genes in each group. In Fig 2, we provide the histograms of the mean Count Per Million (CPM) for the 1000 most stably expressed genes identified in each group. For each gene, the CPM is computed as

$$
\frac{\text { count } \times 10^{6}}{\text { normalized library size }}
$$

in each sample and the mean is computed over all samples.

The lists of the top 1000 genes in the three groups share 104 genes in common (see Supplemental Table S5 for more details). These genes are stably expressed under a wide range of experimental conditions and in different tissue types, and thus may be worth further study. This list of 104 genes has significant overlap with the top 100 stably expressed genes identified by Czechowski et al. from a developmental series of microarray samples: 9 out of these 104 genes (see Supplemental Table S6 for details), 

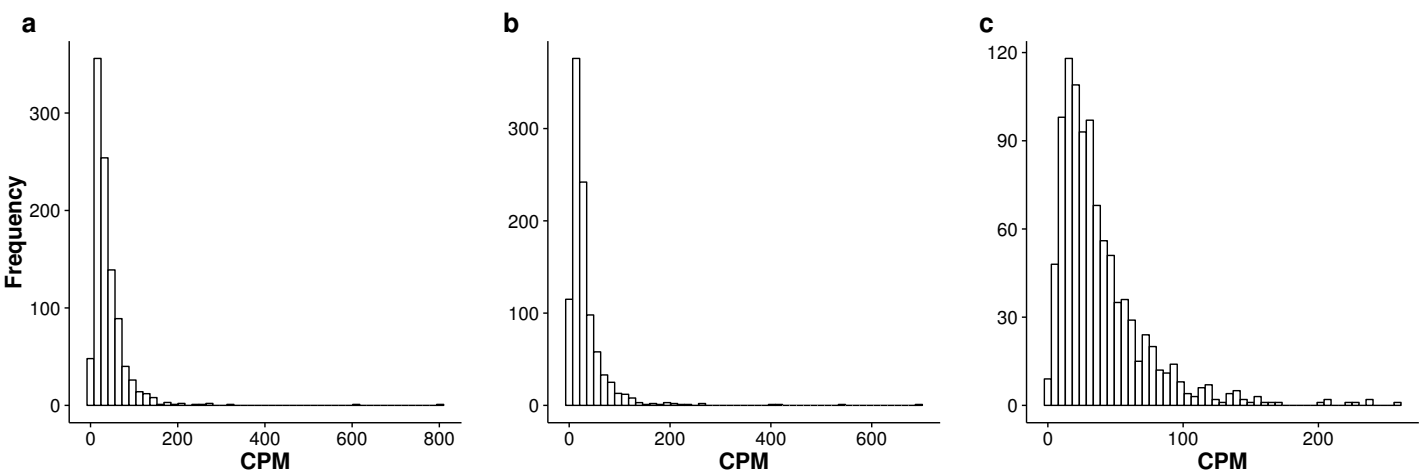

Figure 2. Histograms of the mean CPM (see equation (9)) for the top 1000 most stably expressed genes identified from the seedling (a), leaf (b) and multi-tissue (c) groups using the total variance measure $\hat{\sigma}^{2}$. The mean CPM is computed over all samples within each respective group. Note that the $x$ and $y$ axis scales differ between the three plots.

\section{AT1G13320, AT1G54080, AT2G20790, AT2G32170, AT3G10330, AT4G24550, AT5G26760, AT5G46210, AT5G46630,}

appeared in the list of the top 100 stably expressed genes out of 14000 genes they examined (the probability is $4.8 \times 10^{-9}$ for a list of 104 genes random selected from a set of 14000 genes to have an overlap of size 9 or more with a pre-selected list of 100 genes). In particular, one gene, AT1G13320, is in all but one of the ten lists of top 500 stably expressed genes identified by Czechowski et al. for different experimental and experimental conditions (the only exception is the set of diurnal series), and is also identified by Hong et al. (2010) as a stably expressed gene under all but one of the six experimental conditions they examined. This gene is ranked 159 (top 0.7\%), 112 (top 0.5\%), 513 (top 2.2\%) in the three groups we examined, respectively, according to our stability measure. This gene is a subunit of protein phosphatase type $2 \mathrm{~A}$ complex and is involved in regulation of phosphorylation and regulation of protein phosphatase type $2 \mathrm{~A}$ activity. It has been used as a reference gene for normalization in many papers (e.g., Baron et al. (2012); Bournier et al. (2013); these two papers cited Czechowski et al. as reference).

\subsection{Comparison to house-keeping genes and stably expressed genes identified from microarray data}

Czechowski et al. discussed the expression stability of house-keeping genes and showed that the housekeeping genes are not stably expressed according to their numerical measure. In particular, they compared the expression profiles of five traditional house-keeping genes (AT1G13440, AT3G18780, AT4G05320, AT5G12250, AT5G60390) and five genes (AT1G13320, AT5G59830, AT2G28390, AT4G33380 and AT4G34270) that they identified as stably expressed according to the CV measure from a developmental series of microarray experiments (see Fig.1 of that paper). In Fig.3, we compare the expression profiles of these 10 genes from Czechowski et al. to the expression profiles of five genes (AT1G64840, AT1G75420, AT2G32910, AT3G51310, AT5G48340) that we randomly selected from the top 100 most stably expressed genes identified from the multi-tissue group RNA-Seq data according the total variance $\hat{\sigma}^{2}$. For each of the 15 genes, Fig. 3 shows the expression levels measured in CPM over 91 samples in the eight experiments in the multi-tissue group, and Table 2 summarizes the variance components estimated from the GLMM in Section 2.3.

The five house-keeping genes show large total variation with all three variance-components relatively large as compared to the other 10 genes. This is consistent with Czechowski's observation that housekeeping genes are not necessarily stably expressed according to a numerical measure. Three of the five stably-expressed genes identified by Czechowski are among the top 1000 stably-expressed genes according to our stability measure, the total variance $\hat{\sigma}^{2}$. Czechowski et al. identified those five genes from microarray data and different experiments. It is not too surprising those genes might not be the most stable in RNA-Seq experiments: the two technologies differ in many aspects including coverage and sensitivity. 


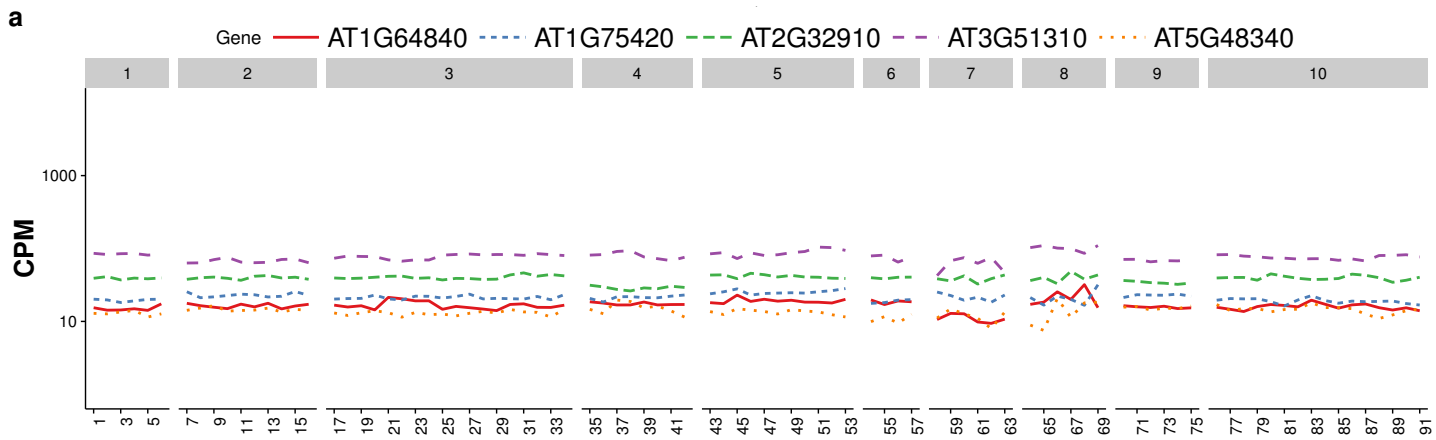

b

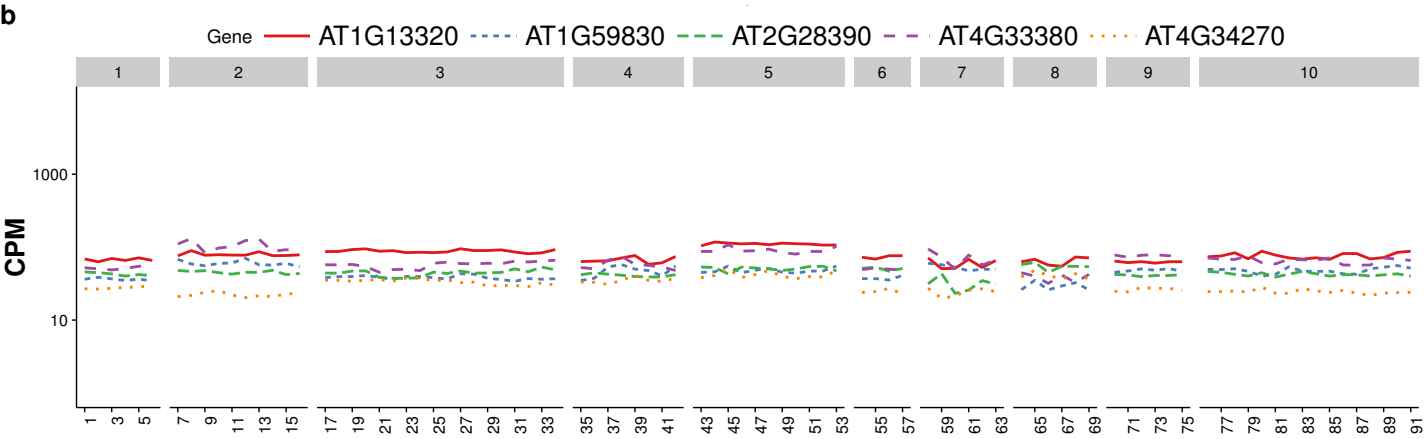

C

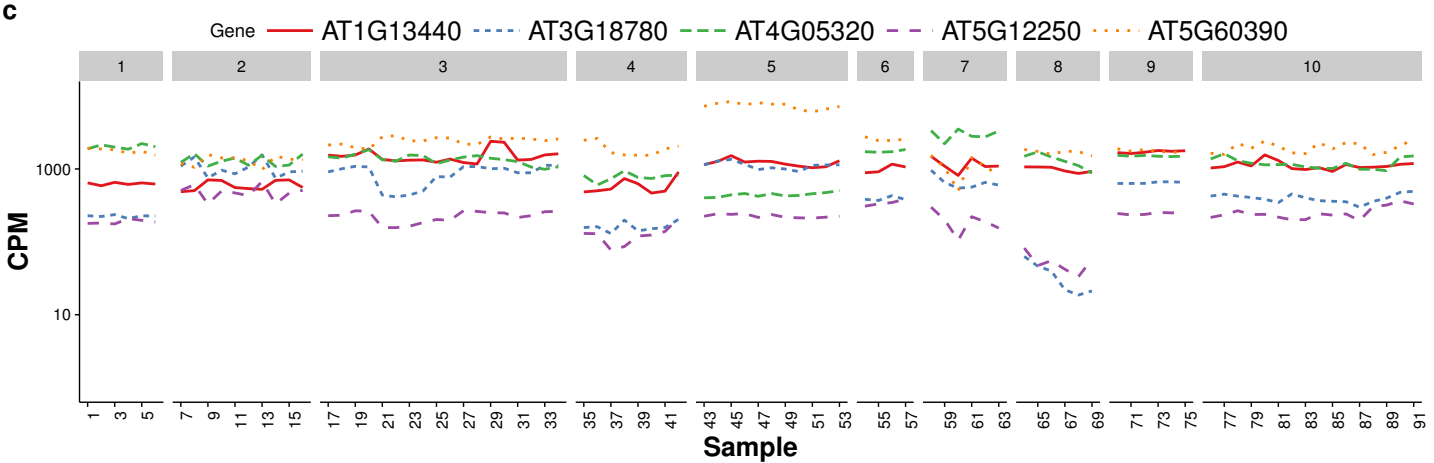

Figure 3. Expression profiles of 15 genes-as measured by RNA-Seq CPM-across 91 samples in the multi-tissue group. The 15 genes include (from top to bottom) (a) five stably expressed genes (randomly selected out of the top 100) identified from the multi-tissue group RNA-Seq data using the total variance measure $\hat{\sigma}^{2}$, (b) five stably expressed identified by Czechowski et al. according to the CV measure from a developmental series of microarray experiments, and (c) five traditional house-keeping genes (HKG) discussed in Czechowski et al.. 
Table 2. Variance components estimated from the multi-tissue group RNA-Seq data for the 15 genes in Fig.3 (identified from different sources). Columns 3-5 are the estimated variance components. Column 6 lists the stability ranking according to the total variance $\hat{\sigma}^{2}$ in the multi-tissue group.

\begin{tabular}{rllllr}
\hline Source & Gene & $\begin{array}{l}\text { betweeen- } \\
\text { sample }\end{array}$ & $\begin{array}{l}\text { between- } \\
\text { treatment }\end{array}$ & $\begin{array}{l}\text { between- } \\
\text { experiment }\end{array}$ & Rank \\
\hline \multirow{5}{*}{ RNA-Seq } & AT1G75420 & 0.0012 & 0.0014 & 0.0050 & 5 \\
& AT5G48340 & 0.0042 & 0.0019 & 0.0074 & 46 \\
& AT2G32910 & 0.0007 & 0.0019 & 0.0113 & 53 \\
& AT1G64840 & 0.0051 & 0.0008 & 0.0095 & 72 \\
& AT3G51310 & 0.0028 & 0.0025 & 0.0100 & 73 \\
\hline Microarray & AT2G28390 & 0.0034 & 0.0000 & 0.0111 & 62 \\
& AT1G13320 & 0.0036 & 0.0003 & 0.0258 & 513 \\
& AT4G34270 & 0.0063 & 0.0000 & 0.0365 & 1074 \\
& AT1G59830 & 0.0044 & 0.0039 & 0.0370 & 1211 \\
& AT4G33380 & 0.0103 & 0.0016 & 0.0747 & 3404 \\
\hline & AT1G13440 & 0.0234 & 0.0058 & 0.1375 & 6562 \\
& AT5G60390 & 0.0267 & 0.0068 & 0.2270 & 8867 \\
HKG & AT4G05320 & 0.0123 & 0.0094 & 0.2690 & 9409 \\
& AT5G12250 & 0.0313 & 0.0128 & 0.3262 & 10589 \\
& AT3G18780 & 0.0375 & 0.0211 & 1.0313 & 14951 \\
\hline
\end{tabular}

The house-keeping genes identified in Czechowski et al. tend to have higher CPM. This is partly due to a selection preference: the authors there intentionally found genes with higher CPM for use as references so that they can be observed in most of the experiments. As we will explain later, we suggest using a collection of 100 to 1000 genes as reference gene set for normalization, we did not specifically target for genes with high CPM.

\subsection{Factors affecting stability ranking}

The previous two subsections demonstrate that when using a numerical measure to quantify gene expression stability, the outcome is dependent on 1) the biological context reflected in the reference sample set used and 2) the technology used for measuring gene expression. It should also be intuitive, and we will further clarify in the second half of this subsection, that the stability ranking is also dependent on 3) the specific numerical measure used. In this section, we will first compare the lists of stably-expressed genes identified under different scenarios where one or more of the above three factors differ. We then further discuss the subtle roles played by the specific stability measure and the reference gene set by comparing the total variance $\hat{\sigma}^{2}$ measure from the GLMM (see equation (6)) to the $M$-value measure used in the geNorm method (Vandesompele et al., 2002).

We look at an additional five lists of stably expressed genes identified under different scenarios and examine how each of these five lists overlaps with the the top stably-expressed genes identified from the multi-tissue group of RNA-Seq experiments according to the total variance measure $\hat{\sigma}^{2}$ (see Section 2.3). The five lists are:

$L_{1}: 100$ top stably expressed genes from the multi-tissue group according to the $M$-value in geNorm (applied to (count +1$)$ ) of Vandesompele et al. ;

$L_{2}: 100$ top stably expressed genes from the seedling group according to the total variance $\hat{\sigma}^{2}$ from the GLMM;

$L_{3}$ : 100 top stably expressed genes from the leaf group according to the total variance $\hat{\sigma}^{2}$ from the GLMM;

$L_{4}: 100$ stably expressed genes identified from a developmental series of microarray experiments by Czechowski et al. using the CV measure (see Section 2.4); 
$L_{5}$ : 50 stably expressed genes identified by Dekkers et al. from microarray seed experiments using the CV measure.

For each list $L_{i}$ above, we measure how it overlaps with the top stably expressed genes (the reference set) from the multi-tissue group using the recall percentage

$$
\frac{\#\left\{L_{i} \cap \text { reference set }\right\}}{\#\left\{L_{i}\right\}} \times 100,
$$

where $\#\{\}$ denotes the number of elements in the list. In Fig.4, we plot the recall percentage versus the number of top stably-expressed genes we selected as reference from the multi-tissue group.

We have the following observations:

1. The list $L_{1}$ is identified from the same set of RNA-Seq experiments as the reference sets, but using a different stability measure ( $M$-value in geNorm). This list has significant overlap with the top stably-expressed genes identified using the total variance measure: 29 and 98 out of the 100 genes from the list $L_{1}$ are among the top 100 and 1000 most stably-expressed genes, respectively, from the multi-tissue group identified using the total variance measure.

2. The lists $L_{2}$ and $L_{3}$ are identified from different sets of RNA-Seq experiments (leaf and seedling experiments) using the same stability measure as used for the reference sets. The lists $L_{4}$ and $L_{5}$ are identified from microarray experiments (a developmental series and a seed group) and using the $\mathrm{CV}$ measure. The overlapping (recall) percentages are still statistically significant, but much less than in the case of $L_{1}$. This shows that differences in tissue type and in measuring technology both influence the expression stability ranking, and to comparable degrees. The lists $L_{3}$ and $L_{5}$ have the least overlapping percentages with the reference sets. These lists are identified from a leaf group and a seed group respectively. Our understanding is that the leaf group and the seed group are more biologically homogeneous than the multi-tissue group and thus provide very different biological contexts for evaluating expression stability.

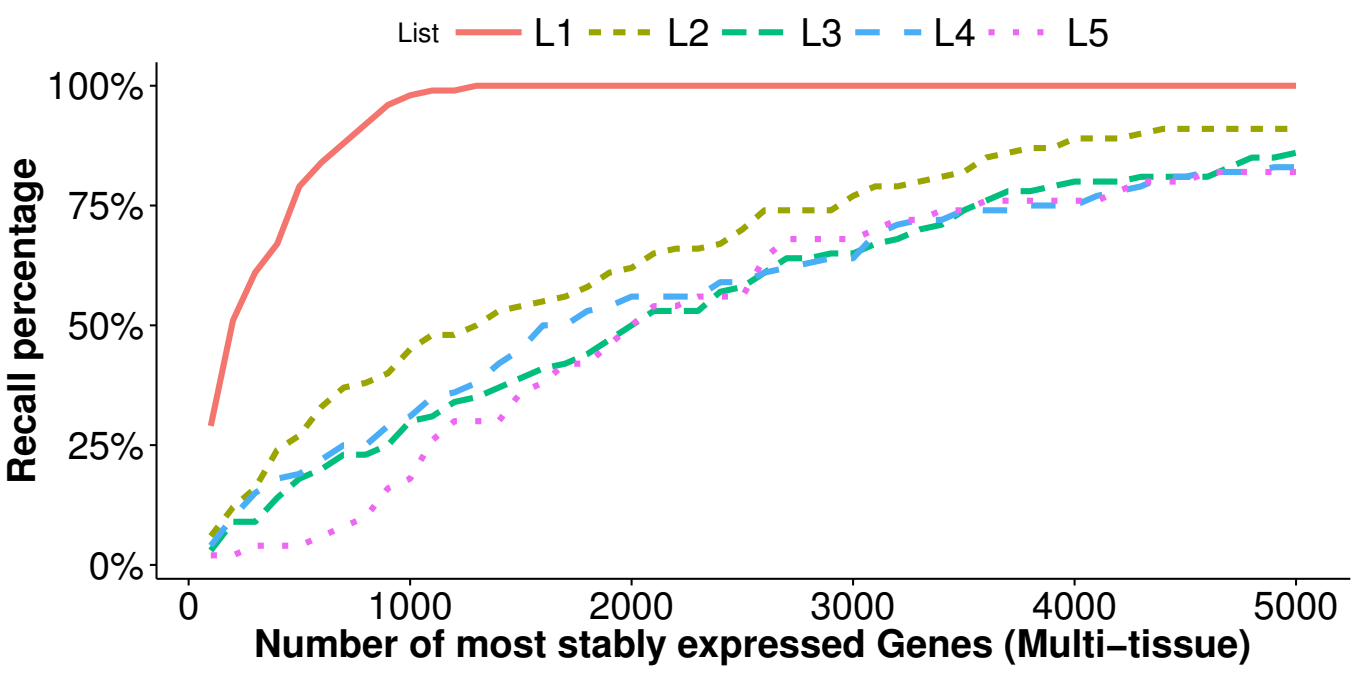

Figure 4. Comparison of top stably expressed genes identified under different scenarios. We choose the top 100 stably expressed genes as described in $L_{1}-L_{4}$, and the top 50 stably expressed genes in $L_{5}$ (see Section 3.3). and plot the recall percentages between these lists and the top most stably expressed genes identified from the multi-tissue group according to the total variance measure. The $x$-axis is the number of most stably expressed genes in multi-tissue group according to the total variance measure, and the $y$-axis shows the recall percentage (see equation (10)) for each of the five lists.

When applied to the same set of samples, the $M$-value and total variance measure $\hat{\sigma}^{2}$ give similar expression stability ranking: the rank correlation is 0.97 (see also, observation 1 above). We point out that 
the reason is because the $M$-value and normalization step needed for computing our total variance measure have similar fundamental assumptions. The basic principle behind the $M$-value is that the expression ratio of two stably-expressed genes should be identical in all samples. In formula, it means that the expression values of two stably-expressed genes $i_{1}, i_{2}$ in any two samples $j_{1}, j_{2}$ should satisfy

$$
\frac{y_{i_{1}, j_{1}}}{y_{i_{2}, j_{1}}}=\frac{y_{i_{1}, j_{2}}}{y_{i_{2}, j_{2}}} .
$$

Our total variance measure $\hat{\sigma}^{2}$ is estimated from normalized data. The basic assumption in the normalization step is that majority of genes are not DE. In formula, it means for any stably-expressed gene $i_{1}$, its expression level as measured by the relative frequency should be stable across all samples,

$$
\frac{y_{i_{1}, j_{1}}}{S_{j_{1}}}=\frac{y_{i_{1}, j_{2}}}{S_{j_{2}}},
$$

where $S_{j_{1}}$ to $S_{j_{2}}$ are the normalized library sizes (i.e., $R_{j} N_{j}$ in equation (6)). This implies for any two stably-expressed genes $i_{1}$ and $i_{2}$

$$
\frac{y_{i_{1}, j_{1}}}{y_{i_{1}, j_{2}}}=\frac{y_{i_{2}, j_{1}}}{y_{i_{2}, j_{2}}}=\frac{S_{j_{1}}}{S_{j_{2}}} .
$$

The first equation in (13) is equivalent to equation (11). (In practical application of both methods, the stability of any single gene is evaluated by comparing its expression to a set of reference genes. See the Method section 2.2 for more details.)

In practice, the geNorm program (Vandesompele et al., 2002) is frequently used to rank a set of reference genes identified from other methods. An iterative elimination procedure is used along with the $M$-value to determine the final ranks of the expression stability: after each iteration, the gene receiving the largest $M$-value will be removed and a new set of $M$-values will be computed for the remaining genes, and the iteration will go on until there are only two genes left. We did not use such an iterative procedure in the comparisons above (i.e., we only computed one set of $M$-values for all genes). We provided some comments about the iterative elimination procedure in the Appendix.

\subsection{Sources of variation}

For each gene, the GLMM (equation (6) of section 2.3) allows us to decompose total count variance into between-sample, between-treatment and between-experiment variance components. The estimated variance components tell us how much each component contributes to the overall count variation. Table 3 summarizes the percentages - averaged over all genes - of the total variance attributable to each of the three components for three groups of RNA-Seq samples (seedling, leaf and multi-tissue groups in Section 2.1). Figure 5 shows the histograms of the percentages. Figure 6 shows the stacked bar plot of variance components estimated from the multi-tissue group for 20 genes randomly selected from the top 1000 stably expressed genes and 20 genes randomly selected from 23611 genes. As expected, the between-experiment variance component, on average, explains the largest proportion of the total variation. The between-experiment variation is relatively smaller among the leaf samples, indicating that the leaf samples are more homogeneous. There is more variation in the relative percentages of total variance explained by the between-sample and between-treatment variance components. In principle, the between-treatment variation will be greater when there is a higher proportion of DE genes or when the samples are more homogeneous. In practice, the between-sample variance depends greatly on what samples are used as biological replicates.

\subsection{Reference gene set for normalization}

Once we have ranked the genes according to our numerical stability measure (i.e, the total variance measure, $\hat{\sigma}^{2}$ ), one application is to use an explicit set of most stably expressed genes as reference genes for count normalization. This new approach allows investigators to prescribe a specific biological context for evaluating gene stability by choosing the most relevant reference samples and experiments when computing the stability measure. For example, the most stably expressed genes identified from the 
Table 3. Percentages — averaged over all genes_ - of the total variance attributable to each of the three variance components (between-sample, between-treatment, between-experiment) for the three groups of RNA-Seq samples (the seedling, the leaf and the multi-tissue groups).

\begin{tabular}{llll}
\hline Source & Seedling & Leaf & Multi-tissue \\
\hline between-sample & $7.2 \%$ & $16.0 \%$ & $7.6 \%$ \\
between-treatment & $20.1 \%$ & $28.0 \%$ & $5.1 \%$ \\
between-experiment & $72.6 \%$ & $56.0 \%$ & $87.3 \%$ \\
\hline
\end{tabular}
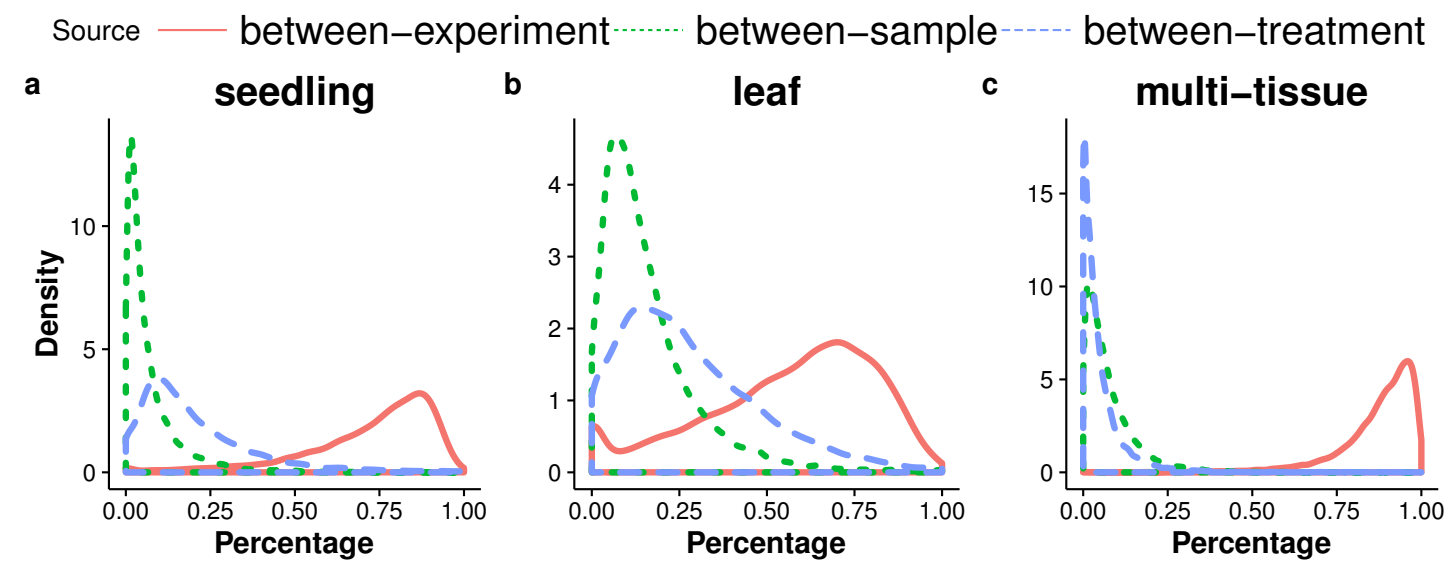

Figure 5. Distributions (over all genes) of the percentages of the total variance attributable to the between-sample, and between-treatment, or the between-experiment variance component, in the seedling (a), the leaf (b), and the multi-tissue groups (c).

multi-tissue group and those identified from the seedling group will provide different biological contexts. In contrast, existing normalization approaches are often applied to the single data set under study, and thus provide a single, narrow context.

Even under a specific biological context, it is almost impossible to know whether the genes in any reference set are absolutely stably expressed, even though commonly used normalization methods often enforce some assumptions on the reference gene set: for example, when we use Anders and Huber's method to estimate the normalization factors based on a subset of reference genes, roughly speaking, the median fold change among the reference genes will be set to 1 (see Section 2.2 for more details). A subtle point we want to make is that since it is impossible to know how well such or similar assumptions on DE hold for a reference gene set, we can improve the interpretability of the DE test results by making the reference gene set explicit: we can slightly change our perspective and interpret all DE results as relative to the reference gene set. For example, a fold change of 2 inferred from the GLMM model can be interpreted as the fold change of a gene is 2 times the true (but often unknowable) median fold change of the reference genes. When one estimates the normalization factors based on all genes, one is effectively specifying an implicit set of genes as a reference set. Our proposal is to make the reference set explicit and interpret DE results as relative to the reference gene set.

Interpreting the DE results as relative to an explicit reference set is especially beneficial when one wants to compare DE results from an experiment to previously published results. When the interest is in comparing different experiments, we recommend using a common reference set. For example, when two RNA-Seq data sets are separately normalized with different reference sets, a fold change of two observed in one experiment may not be directly comparable to a fold change of two observed in the other. This concern can be alleviated by using a common set of reference genes. We use a toy example to illustrate this point in Table 4 where we examine the mean counts for 5 genes in two two-group comparison experiments. If we use different reference gene sets for count normalization in the two experiments, for 


\section{Source $\square$ sample $\square$ treatment $₫$ experiment}
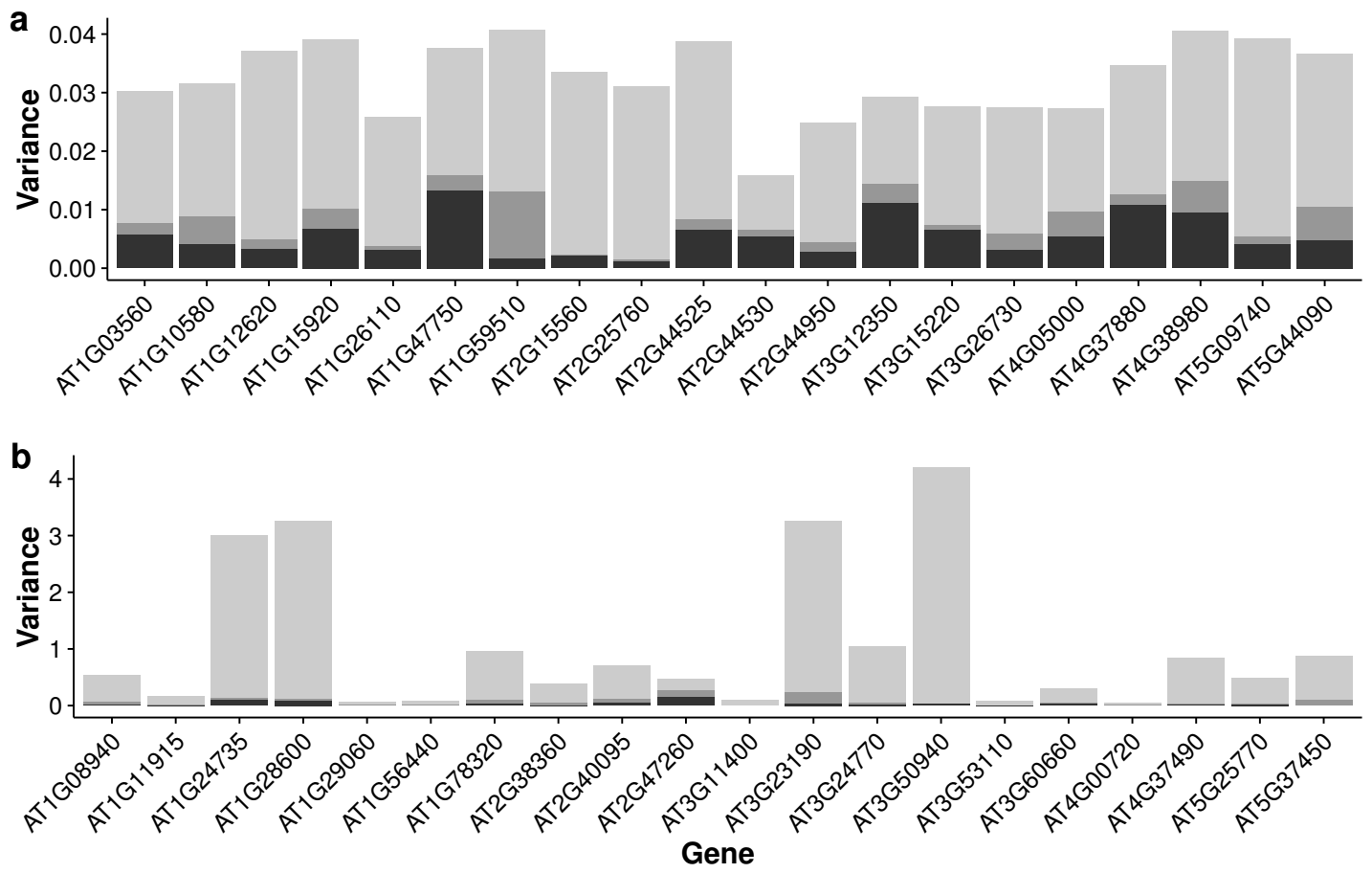

Figure 6. Stacked bar plots of the three variance components for selected genes in the multi-tissue group. (a): 20 genes randomly selected from top 1000 stably expressed genes; (b): 20 genes randomly selected from all the genes.

example, we use genes 1-3 as reference in experiment 1, but use genes 3-5 as reference in experiment 2 , we may conclude that gene 3 is not DE in either experiment. If we use a common reference gene set-either genes 1-3 or genes 3-5-for normalization, however, we will be able to discover, in either case, that the DE behavior of gene 3 is different in the two experiments. Note that the DE conclusion in both experiments will depend on the reference genes used: if genes 1-3 are used as reference, gene 3 is not DE in experiment 1, but will be DE in experiment 2; if genes 3-5 are used as reference, gene 3 will be considered DE in experiment 1 , but not DE in experiment 2 . The point is, in either case, we will notice that the DE behavior of gene 3 is different between the two experiments. This information will be lost if one uses different reference sets to assess DE in the two experiments.

In practice, we recommend using the top 1000 most stably expressed genes for estimating normalization factors. The key is to avoid using too few (e.g., less than 10) or too many (e.g., using all genes) reference genes: intuitively, using too few, the estimates will be unstable; using too many, the results may be subject to influence from highly unstable genes. Our simple simulations suggest that using between 100 to 10000 genes seems to give stable results. In the first set of three examples, we used Anders and Huber's method (see equation (2)) to estimate normalization factors for samples in each of the seedling, leaf and multi-tissue groups of experiments (see Section 2.1). We used the top 10, 100, 1000, and 10000 stably expressed genes identified earlier (see Section 3.1 for details) as reference gene sets. Figure 7 shows the pairwise scatter plots and correlation coefficient between the normalization factors when different numbers of top stable genes are used as reference. A stronger correlation indicates the normalization factors estimated from the two settings are highly consistent. The plots and correlation coefficients suggest using between 100 and 1000 genes tend to give similar normalization factor estimates. We also used the top 10,100,1000, and 10000 stably expressed genes identified from the multi-tissue group as reference set for estimating normalization factors for a set of 48 root samples from a new experiment (GSE64410, Vragović et al. (2015)). The largest Pearson correlation 0.993 is between the normalization factors estimated using the top 100 and top 1000 stably expressed genes as reference. Based on the above 
Table 4. A toy example for illustrating the importance of using a common explicit set of reference genes when comparing RNA-Seq data from multiple experiments. If a common reference gene set (e.g., genes $1-3$ ) is used as reference for count normalization, we will notice that the DE behavior of gene 3 differs in the two experiments. If the two experiments are separately normalized using genes $1-3$ as reference in experiment 1 , but using genes $3-5$ as reference in experiment 2 , we may conclude that gene 3 is not DE in either group.

\begin{tabular}{cccccc}
\hline & \multicolumn{2}{c}{ Exp. 1 } & & \multicolumn{2}{c}{ Exp. 2 } \\
\cline { 2 - 3 } \cline { 5 - 6 } Gene & Control & Treatment & & Control & Treatmetn \\
\hline 1 & 10 & 20 & & 10 & 20 \\
2 & 10 & 20 & & 10 & 20 \\
3 & 10 & 20 & & 10 & 10 \\
4 & 10 & 10 & & 10 & 10 \\
5 & 10 & 10 & & 10 & 10 \\
\hline
\end{tabular}

observations, using 1000 most stably expressed genes as reference seems to be a reasonable heuristic rule.

\subsection{An example}

In this part, we illustrate the effect of using different reference gene sets for computing normalization factors on a real data set and explain the implication on DE analysis.

Wang et al. (2012) performed RNA-Seq experiments using 10-day old seedlings to investigate the role of Arabidopsis SNW/Ski-interacting (SKIP) protein on transcriptome-wide changes in alternative splicing. Two biological replicates each from wild type (Col-0) and skip-2 mutant were compared. We retrieved and processed the raw RNA-Seq data from this experiment using our pipeline (see Section 2.1, accession number GSE32216). For this data set, the normalization factors for the four samples (two wild types followed by two mutants) estimated using all genes, $(0.84,0.62,1.38,1.39)$, differ markedly from the normalization factors, $(0.71,0.54,1.59,1.63)$, estimated using the 1000 reference genes that we identified using the total variance measure from the seedling group (see section 3.1).

The implication on DE analysis is that if we use the 1000 stably expressed genes for normalization, we will expect to see more under-expressed genes and less over-expressed gene in the mutant group relative to the wild type group. The two sets of estimated normalization factors reflect different assumptions: roughly speaking, when using all genes to compute the normalization factors, the assumption is that median fold change among all genes is 1 ; when using the 1000 reference genes to compute the normalization factors, the assumption is that the median fold change among the set of 1000 genes is 1 . It is difficult to know which assumption is more reasonable without additional biological insights. However, the benefit of using an explicit set of 1000 genes as reference is the improved interpretability by making the reference gene set, and thus the implied assumption, more explicit. Furthermore, if one wants to compare the DE results from this experiment to other DE results from the collection of seedling experiments, then one should use a common reference set of genes for count normalization.

\section{CONCLUSION AND DISCUSSION}

In this paper, we advocate quantifying gene expression stability by applying a numerical stability measure to a large number of existing RNA-Seq data sets. Similar strategies have also been used by others to find stably expressed genes from microarray data. Since DE is measured by relative frequencies, we argue that $\mathrm{DE}$ is a relative concept and using an explicit reference gene set can improve interpretability of DE results, and furthermore, using a common reference gene set can avoid inconsistent conclusions when comparing multiple experiments (see Section 3.5).

It should be clear but worth emphasizing that when using a numerical measure to identify stably expressed genes, the outcome depends on multiple factors: the background sample set and the reference gene set used for count normalization, the technology used for measuring gene expression, and the specific numerical stability measure used. In this study, to illustrate our proposed methods, we identified three sets of stably expressed genes from three sets of Arabidopsis experiments. The major point is that stably expressed genes identified from different backgrounds will provide different biological contexts for evaluating differential expression. In practice, researchers can choose the specific context. A practical 


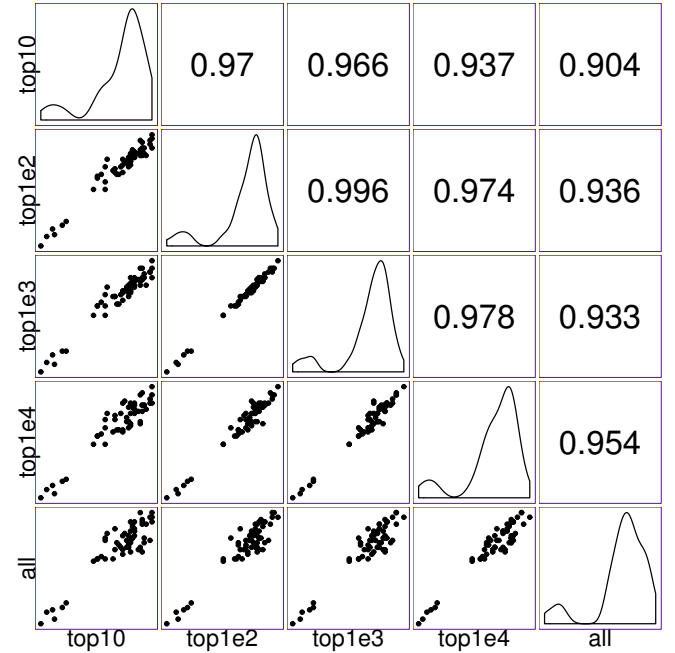

(a)

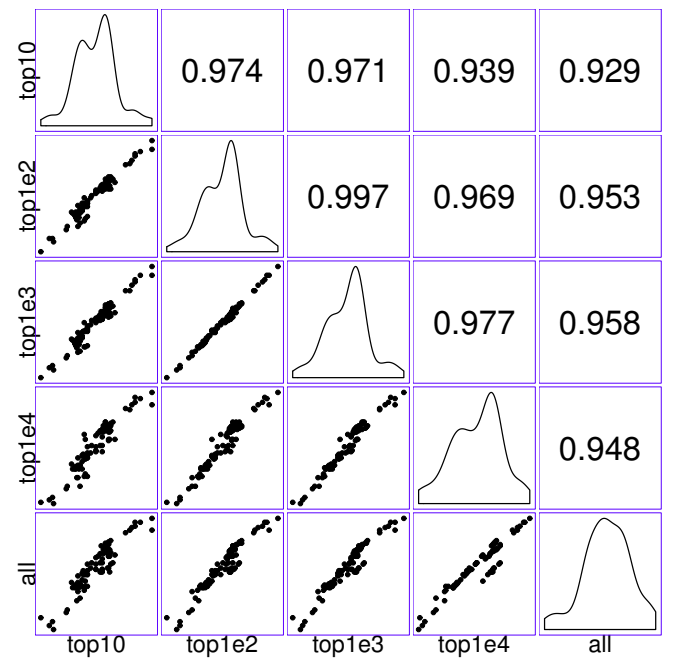

(c)

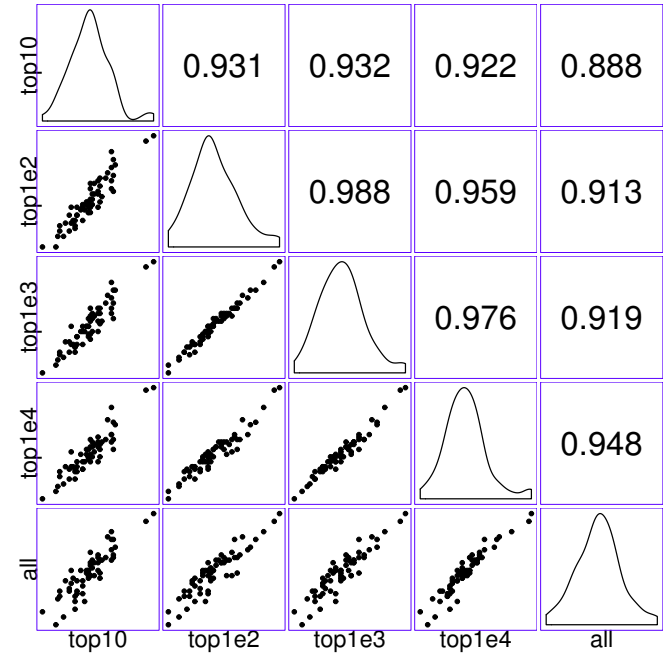

(b)

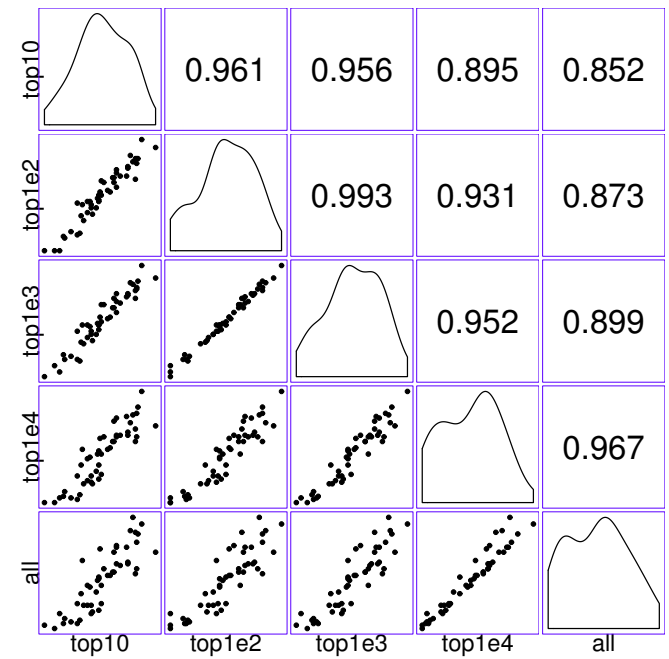

(d)

Figure 7. Matrices of scatter plots of normalization factors estimated using different reference gene sets. The subfigures (a), (b) and (c) show normalization factors estimated for the samples in the seedling, leaf, and multi-tissue groups correspondingly. In each case, the top 10,100, 1000, and 10,000 stably expressed genes are used as reference to calculate the normalization factors. The subfigure (d) shows the normalization factors estimated for a new root experiment (GSE64410, with sample size 48) using the top $10-10,000$ stably expressed genes identified from the multi-tissue group as reference. The normalization factors are estimated using the method described in Section 2.2.

challenge in applying such a philosophy is that no two experiments will have identical settings, and researchers have to decide what experiments can be considered comparable. This is a difficult question; however, we believe it has to be asked from now on: biologists perform comparative experiments with the intent that the conclusions from a single experiment will be generalizable beyond the context of a single lab. If we do not understand comparability between different experiments, such generalization is impossible. Defining and characterizing comparability is a challenging topic that we would like to 
investigate more in the future.

To identify a set of stably expressed genes, our method still needs to estimate an initial set of normalization factors, which requires that we must make assumptions about relative fold changes between samples. This kind of circular dependence seems unavoidable Vandesompele et al.. In this paper, we used a one-step iteration strategy to reduce the dependence on the initially estimated normalization factors. In future work, we intend to look at the genes through evolutionary genetics methods (e.g., 1001 genomes, Weigel and Mott (2009)). For example, evolutionary genetics methods can help us test whether a gene is under negative, neutral, or positive selection and help us identify genes that are well conserved through the evolutionary history. We need to be mindful that a well conserved gene is not necessarily stably expressed, just like the house-keeping genes. However, it would be interesting to ask whether there is correlation between measures of expression stability and measures of conservativeness, and so on.

In the GLMM model we fit, the random effect terms such as the sample and treatment effects were modeled as normal random variables (Section 2.3). For the purpose of identifying stably expressed genes, this should be adequate, since we are mainly interested in the variances of these random effects (i.e., the variance components). In the future, it may also be of interest to model these random effects more accurately, for example, in order to build a prior distribution of the random effect terms for analyzing a new data set. A more careful examination of the individual data sets suggests that the between-sample variance varies greatly between experiments. Our observation suggests that different labs often have different understanding of what is deemed as "biological replicates".

The R codes for reproducing results in this paper are available at Github: https: / / github.com/ zhuob/StablyExpressedGenes

\section{APPENDIX. THE ITERATIVE ELIMINATION PROCEDURE IN GENORM}

In this part, we discuss the effect of an iterative elimination procedure used by geNorm. This iterative elimination procedure creates an extra layer of complexity that is not well explored in literature. We use a toy example below to illustrate one subtle aspect of the iterative elimination procedure. In this example, we consider the expression values of 7 genes in two samples shown in Table 5. When $M$-value is used to rank all 7 genes, the initial ranking of expression stability is given in column 4 of the table: gene 7 is the least stable and genes 4 and 5 are considered the most stable ones. Once genes 6 and 7 are eliminated, however, the recalculated $M$-values will rank genes 1-3 as more stable than genes 4 and 5 (see column 5 of Table 5). The root cause of this reversal of ranking is that when an iterative elimination procedure is used, effectively, the reference gene set is changing after each iteration: in the initial ranking, the expression patterns genes 4 and 5 are close to the "middle of the pack" and thus considered as the most stable, and the expression patterns of genes 1-3 and genes 6 and 7 are considered relatively more extreme; once genes 6 and 7 are removed, however, the "middle of the pack" is shifted towards the expression patterns of genes 1-3, and thus genes 1-3 become the most stably expressed. With this understanding, one could and should make a conscious decision on whether such a behavior as described above is desirable or not.

The essence of the above toy example is that the expression profiles of the set of genes to be ranked are clustered into subgroups. In practice geNorm is often used to rank a set of stably expressed genes. In such applications, the impact of the iterative elimination might be limited. For example, if we use $M$-value to rank the top 1000 stably expressed genes identified from the multi-tissue group (3.1), the top 100 mostly stably expressed genes from geNorm runs with and without using the iterative elimination will have 77 genes in common.

The point we want to emphasize is that gene stability is a relative concept and the stability ranking depends on which set of genes we use as reference. In an iterative elimination procedure, the reference gene set will change after each iteration. The procedure can thus give surprising results and the adoption of it in practice should not be automatic.

\section{ACKNOWLEDGEMENT}

We thank Duo Jiang and Wanli Zhang for helpful discussions. This article is part of doctor dissertation written by BZ under the supervision of YD. 
Table 5. A toy example showing the effect of iterative elimination. Columns 2 and 3 represent expression levels for seven genes in two samples, column 4 is the stability ranking of genes by $M$-value without iterative elimination, and column 5 is the ranking after two geNorm iterations.

\begin{tabular}{rrlrl}
\hline & \multicolumn{2}{c}{ Raw Counts } & \multicolumn{2}{l}{ Rank } \\
Gene & sample 1 & sample 2 & rank 1 & rank 2 \\
\hline Gene1 & 1 & 1 & 3 & 1 \\
Gene2 & 1 & 1 & 3 & 1 \\
Gene3 & 1 & 1 & 3 & 1 \\
Gene4 & 1 & 2 & 1 & 4 \\
Gene5 & 1 & 2 & 1 & 4 \\
Gene6 & 1 & 3 & 6 & \\
Gene7 & 1 & 4 & 7 & \\
\hline Library Size & 7 & 14 & & \\
\hline
\end{tabular}

\section{REFERENCES}

Anders, S. and Huber, W. (2010). Differential expression analysis for sequence count data. Genome Biology, 11(10):R106.

Anders, S., McCarthy, D. J., Chen, Y., Okoniewski, M., Smyth, G. K., Huber, W., and Robinson, M. D. (2013). Count-based differential expression analysis of RNA sequencing data using R and Bioconductor. Nature Protocols, 8(9):1765-1786.

Anders, S., Pyl, P. T., and Huber, W. (2014). HTSeq-a Python framework to work with high-throughput sequencing data. Bioinformatics, page btu638.

Andersen, C. L., Jensen, J. L., and Ørntoft, T. F. (2004). Normalization of real-time quantitative reverse transcription-PCR data: a model-based variance estimation approach to identify genes suited for normalization, applied to bladder and colon cancer data sets. Cancer Research, 64(15):5245-5250.

Baron, K. N., Schroeder, D. F., and Stasolla, C. (2012). Transcriptional response of abscisic acid (ABA) metabolism and transport to cold and heat stress applied at the reproductive stage of development in Arabidopsis thaliana. Plant Science, 188:48-59.

Bates, D., Maechler, M., and Bolker, B. (2012). lme4: Linear mixed-effects models using S4 classes.

Bournier, M., Tissot, N., Mari, S., Boucherez, J., Lacombe, E., Briat, J.-F., and Gaymard, F. (2013). Arabidopsis ferritin 1 (AtFer1) gene regulation by the phosphate starvation response 1 (AtPHR1) transcription factor reveals a direct molecular link between iron and phosphate homeostasis. Journal of Biological Chemistry, 288(31):22670-22680.

Bustin, S. (2002). Quantification of mRNA using real-time reverse transcription PCR (RT-PCR): trends and problems. Journal of Molecular Endocrinology, 29(1):23-39.

Czechowski, T., Stitt, M., Altmann, T., Udvardi, M. K., and Scheible, W.-R. (2005). Genome-wide identification and testing of superior reference genes for transcript normalization in Arabidopsis. Plant Physiology, 139(1):5-17.

Dekkers, B. J., Willems, L., Bassel, G. W., van Bolderen-Veldkamp, R. M., Ligterink, W., Hilhorst, H. W., and Bentsink, L. (2012). Identification of reference genes for RT-qPCR expression analysis in Arabidopsis and tomato seeds. Plant and Cell Physiology, 53(1):28-37.

Di, Y., Schafer, D. W., Cumbie, J. S., and Chang, J. H. (2011). The NBP negative binomial model for assessing differential gene expression from RNA-Seq. Statistical Applications in Genetics and Molecular Biology, 10(1):1-28.

Di, Y., Schafer, D. W., and Di, M. Y. (2014). Package 'NBPSeq'. Molecular Biology, 10:1. 
Fernandes, J. M., Mommens, M., Hagen, Ø., Babiak, I., and Solberg, C. (2008). Selection of suitable reference genes for real-time PCR studies of Atlantic halibut development. Comparative Biochemistry and Physiology Part B: Biochemistry and Molecular Biology, 150(1):23-32.

Frericks, M. and Esser, C. (2008). A toolbox of novel murine house-keeping genes identified by metaanalysis of large scale gene expression profiles. Biochimica et Biophysica Acta (BBA)-Gene Regulatory Mechanisms, 1779(12):830-837.

Gur-Dedeoglu, B., Konu, O., Bozkurt, B., Ergul, G., Seckin, S., and Yulug, I. G. (2009). Identification of endogenous reference genes for qRT-PCR analysis in normal matched breast tumor tissues. Oncology Research Featuring Preclinical and Clinical Cancer Therapeutics, 17(8):353-365.

Hong, S. M., Bahn, S. C., Lyu, A., Jung, H. S., and Ahn, J. H. (2010). Identification and testing of superior reference genes for a starting pool of transcript normalization in Arabidopsis. Plant and Cell Physiology, 51(10):1694-1706.

Hruz, T., Wyss, M., Docquier, M., Pfaffl, M. W., Masanetz, S., Borghi, L., Verbrugghe, P., Kalaydjieva, L., Bleuler, S., Laule, O., Descombes, P., Gruissem, W., and Zimmermann, P. (2011). RefGenes: identification of reliable and condition specific reference genes for RT-qPCR data normalization. BMC Genomics, 12(1):156.

Huggett, J., Dheda, K., Bustin, S., and Zumla, A. (2005). Real-time RT-PCR normalisation; strategies and considerations. Genes and Immunity, 6(4):279-284.

Langmead, B. and Salzberg, S. L. (2012). Fast gapped-read alignment with Bowtie 2. Nature Methods, 9(4):357-359.

Leinonen, R., Sugawara, H., and Shumway, M. (2010). The sequence read archive. Nucleic Acids Research, page gkq1019.

Li, H. and Durbin, R. (2009). Fast and accurate short read alignment with Burrows-Wheeler transform. Bioinformatics, 25(14):1754-1760.

Liao, Y., Smyth, G. K., and Shi, W. (2013). The Subread aligner: fast, accurate and scalable read mapping by seed-and-vote. Nucleic Acids Research, 41(10):e108-e108.

Lovén, J., Orlando, D. A., Sigova, A. A., Lin, C. Y., Rahl, P. B., Burge, C. B., Levens, D. L., Lee, T. I., and Young, R. A. (2012). Revisiting global gene expression analysis. Cell, 151(3):476-482.

McCulloch, C. E. and Neuhaus, J. M. (2001). Generalized linear mixed models. Wiley Online Library.

R Core Team (2015). R: A Language and Environment for Statistical Computing. R Foundation for Statistical Computing, Vienna, Austria.

Reid, K. E., Olsson, N., Schlosser, J., Peng, F., and Lund, S. T. (2006). An optimized grapevine RNA isolation procedure and statistical determination of reference genes for real-time RT-PCR during berry development. BMC Plant Biology, 6(1):27.

Risso, D., Ngai, J., Speed, T. P., and Dudoit, S. (2014). Normalization of RNA-seq data using factor analysis of control genes or samples. Nature Biotechnology, 32(9):896-902.

Robinson, M. D. and Oshlack, A. (2010). A scaling normalization method for differential expression analysis of RNA-seq data. Genome Biology, 11(3):R25.

Stamova, B. S., Apperson, M., Walker, W. L., Tian, Y., Xu, H., Adamczy, P., Zhan, X., Liu, D.-Z., Ander, B. P., Liao, I. H., Gregg, J. P., Turner, R. J., Jickling, G., Lit, L., and Sharp, F. R. (2009). Identification and validation of suitable endogenous reference genes for gene expression studies in human peripheral blood. BMC Medical Genomics, 2(1):49.

Vandesompele, J., De Preter, K., Pattyn, F., Poppe, B., Van Roy, N., De Paepe, A., and Speleman, F. (2002). Accurate normalization of real-time quantitative RT-PCR data by geometric averaging of multiple internal control genes. Genome Biology, 3(7):research0034. 
Vragović, K., Sela, A., Friedlander-Shani, L., Fridman, Y., Hacham, Y., Holland, N., Bartom, E., Mockler, T. C., and Savaldi-Goldstein, S. (2015). Translatome analyses capture of opposing tissuespecific brassinosteroid signals orchestrating root meristem differentiation. Proceedings of the National Academy of Sciences, 112(3):923-928.

Wang, X., Wu, F., Xie, Q., Wang, H., Wang, Y., Yue, Y., Gahura, O., Ma, S., Liu, L., Cao, Y., Jiao, Y., Puta, F., McClung, R. C., Xu, X., and Ma, L. (2012). SKIP is a component of the spliceosome linking alternative splicing and the circadian clock in Arabidopsis. The Plant Cell, 24(8):3278-3295.

Weigel, D. and Mott, R. (2009). The 1001 genomes project for Arabidopsis thaliana. Genome Biology, $10(5): 1$.

Wu, D., Hu, Y., Tong, S., Williams, B. R., Smyth, G. K., and Gantier, M. P. (2013). The use of miRNA microarrays for the analysis of cancer samples with global miRNA decrease. $R N A, 19(7): 876-888$. 